Supporting Information

\title{
Investigation on Isobaric Vapor-Liquid Equilibrium for the Binary Systems of Ethyl Benzoate with Xylene Isomers and Ethylbenzene
}

Jiahai Ding, Wei Guan, Leiyu Zhang, Xiudong Zhang, Chundong Zhang*, Lei Wang, Hui Wan, Guofeng Guan*

State Key Laboratory of Materials-Oriented Chemical Engineering, College of Chemical Engineering, Nanjing Tech University, Nanjing 210009, P. R. China Corresponding authors:

Chundong Zhang*, email: zhangcd@njtech.edu.cn, tel.: +86-25-5813-8020

Guofeng Guan*, email: guangf@njtech.edu.cn, tel.: +86-25-8358-7198 


\section{Figure captions}

Figure 1 Modified Rose recirculation still

Figure 2 Plot of activity coefficients $\left(\gamma_{1}, \gamma_{2}\right)$ and molar fraction in liquid phase $\left(x_{1}\right)$ for the system ethylbenzene (1) + ethyl benzoate (2) at $101.33 \mathrm{kPa}$ : Experimental $\gamma_{1}-x_{1} ;-$, correlated $\gamma_{1}-x_{1}$ using the NRTL equation; $\circ$, Experimental $\gamma_{2}-x_{1} ; \ldots$, correlated $\gamma_{2}-x_{1}$ using the NRTL equation

Figure 3 Plot of activity coefficients $\left(\gamma_{1}, \gamma_{2}\right)$ and molar fraction in liquid phase $\left(x_{1}\right)$ for the system $o$-xylene (1) + ethyl benzoate (2) at $101.33 \mathrm{kPa}$ : Experimental $\gamma_{1}-x_{1} ;-$, correlated $\gamma_{1}-x_{1}$ using the NRTL equation; $\circ$, Experimental $\gamma_{2}-x_{1} ; \ldots$, correlated $\gamma_{2}-x_{1}$ using the NRTL equation

Figure 4 Plot of activity coefficients $\left(\gamma_{1}, \gamma_{2}\right)$ and molar fraction in liquid phase $\left(x_{1}\right)$ for the system $m$-xylene (1) + ethyl benzoate (2) at $101.33 \mathrm{kPa}$ : Experimental $\gamma_{1}-x_{1} ;-$, correlated $\gamma_{1}-x_{1}$ using the NRTL equation; $\circ$, Experimental $\gamma_{2}-x_{1} ; \ldots$, correlated $\gamma_{2}-x_{1}$ using the NRTL equation

Figure 5 Plot of activity coefficients $\left(\gamma_{1}, \gamma_{2}\right)$ and molar fraction in liquid phase $\left(x_{1}\right)$ for the system $p$-xylene (1) + ethyl benzoate (2) at $101.33 \mathrm{kPa}$ : Experimental $\gamma_{1}-x_{1} ;-$, correlated $\gamma_{1}-x_{1}$ using the NRTL equation; $\circ$, Experimental $\gamma_{2}-x_{1} ; \ldots$, correlated $\gamma_{2}-x_{1}$ using the NRTL equation 


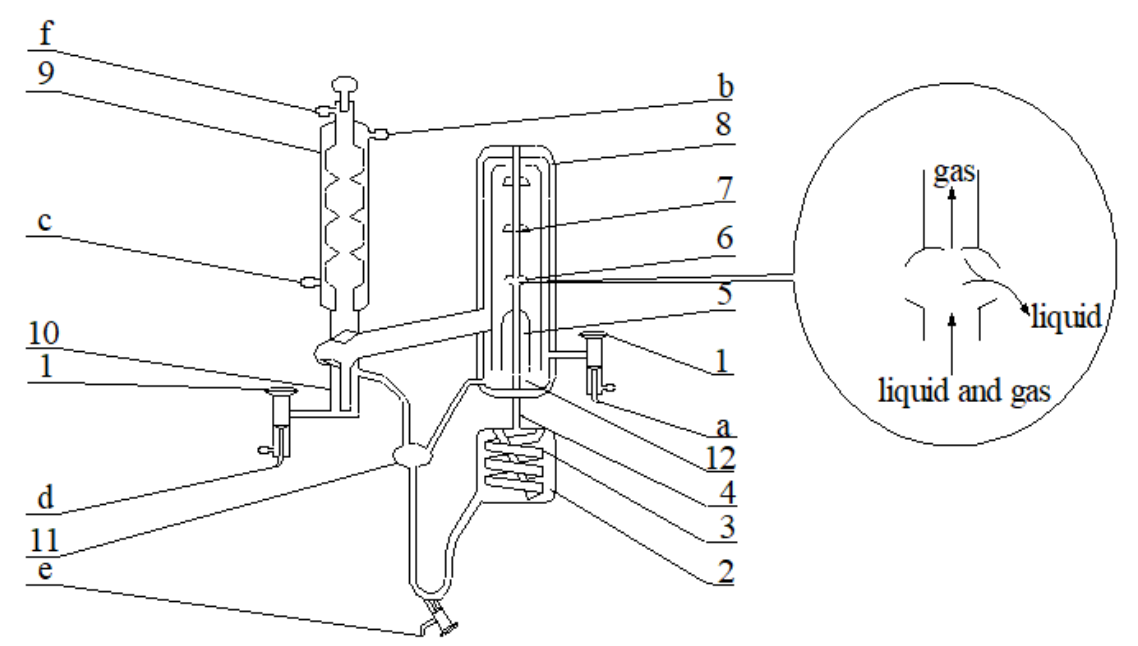

Figure S1. Modified Rose recirculation still, (a) liquid sample connection, (b) cooling water outlet, (c) cooling water inlet, (d) vapor sample connection, (e) liquid discharging outlet, (f) to pressure controlling devices, (1) teflon cock, (2) boiler, (3) inner heater, (4) vapor-liquid riser, (5) gas chamber, (6) vapor-liquid separator, (7) thermometer casing, (8) vacuum thermal insulating jacket, (9) vapor condenser, (10) vapor phase sample reservoir, (11) vapor liquid mixing chamber, (12) liquid phase sample reservoir.

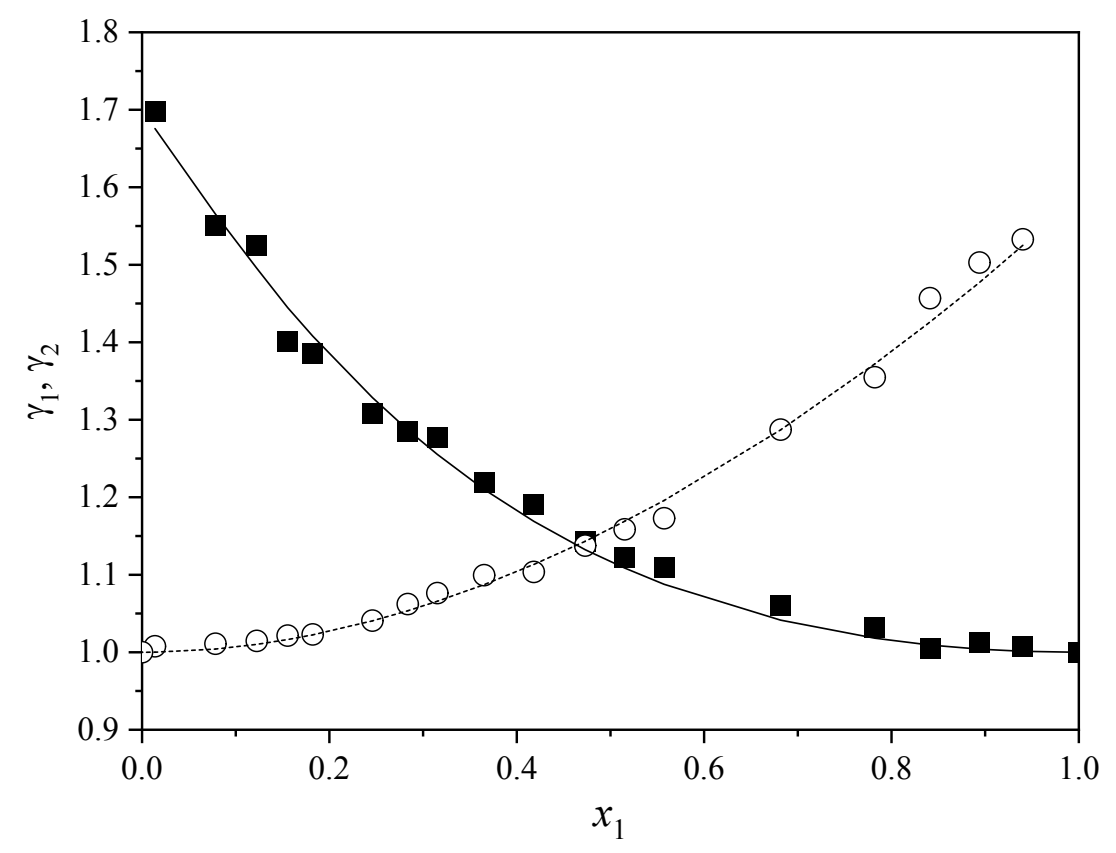

Figure S2. Plot of activity coefficients $\left(\gamma_{1}, \gamma_{2}\right)$ and molar fraction in liquid phase $\left(x_{1}\right)$ for the system ethylbenzene (1)

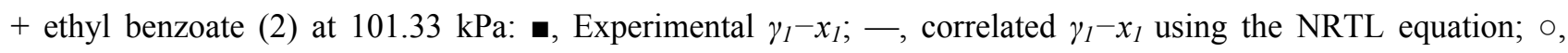
Experimental $\gamma_{2}-x_{1} ; \ldots$, correlated $\gamma_{2}-x_{1}$ using the NRTL equation 


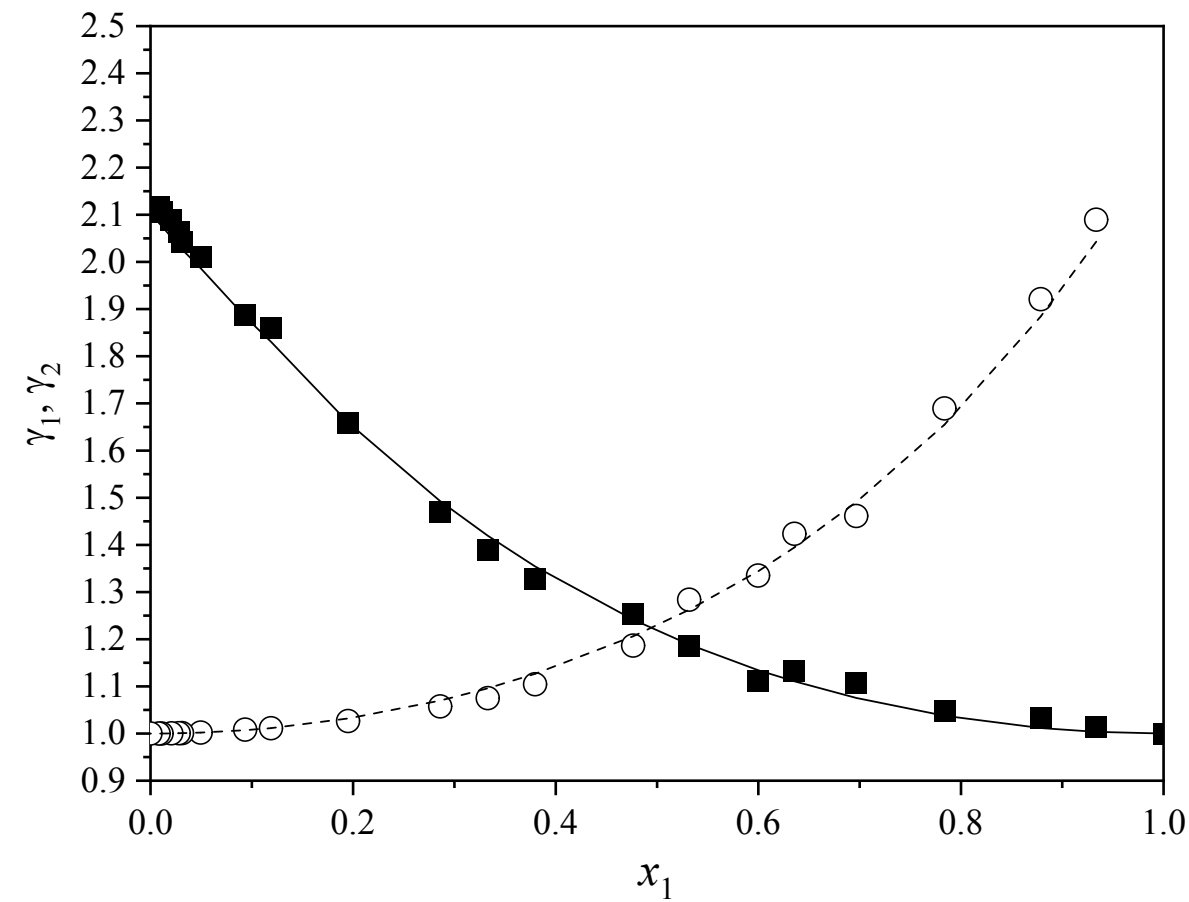

Figure S3. Plot of activity coefficients $\left(\gamma_{1}, \gamma_{2}\right)$ and molar fraction in liquid phase $\left(x_{1}\right)$ for the system $o$-xylene $(1)+$ ethyl benzoate (2) at $101.33 \mathrm{kPa}$ : —, Experimental $\gamma_{1}-x_{1}$; - correlated $\gamma_{1}-x_{1}$ using the NRTL equation; $\circ$, Experimental $\gamma_{2}-x_{1} ; \ldots$, correlated $\gamma_{2}-x_{1}$ using the NRTL equation.

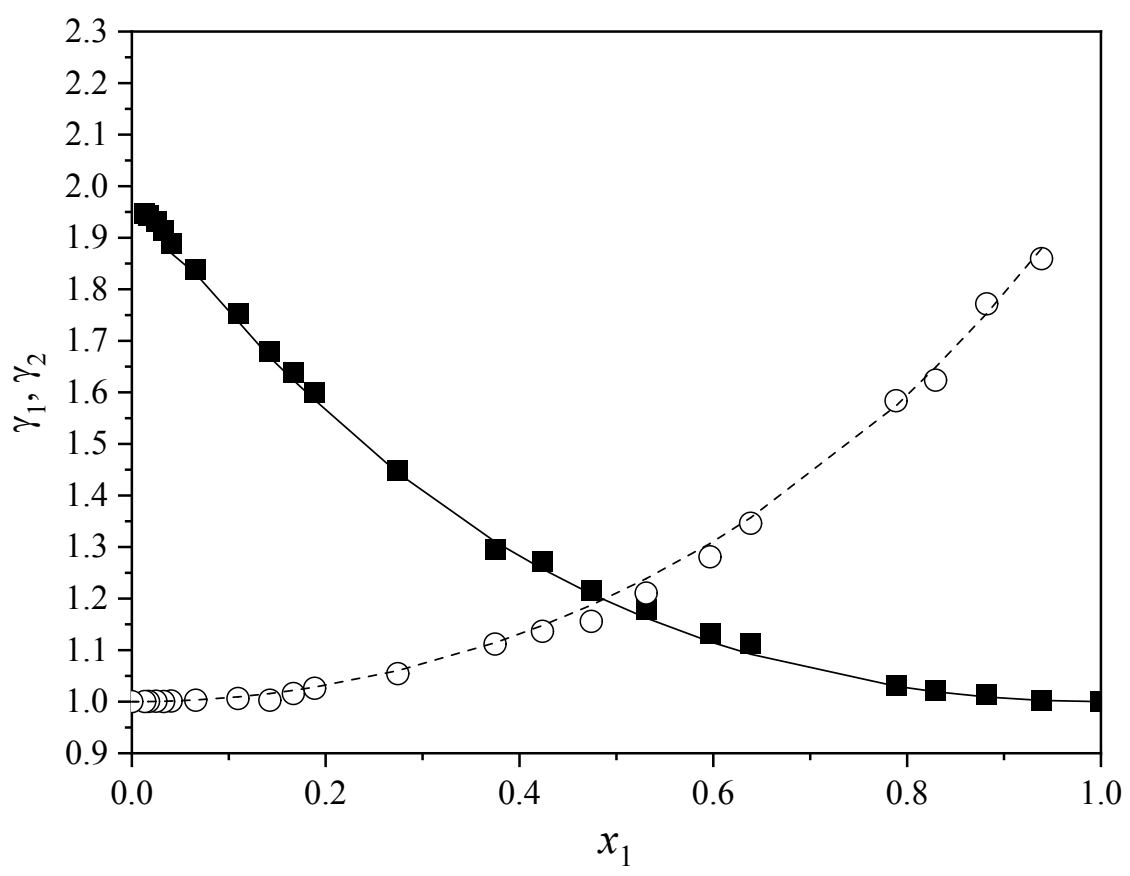

Figure S4. Plot of activity coefficients $\left(\gamma_{1}, \gamma_{2}\right)$ and molar fraction in liquid phase $\left(x_{1}\right)$ for the system $m$-xylene (1) + ethyl benzoate (2) at $101.33 \mathrm{kPa}: \mathbf{m}$, Experimental $\gamma_{1}-x_{1}$; - correlated $\gamma_{1}-x_{1}$ using the NRTL equation; $\circ$, Experimental $\gamma_{2}-x_{1} ; \ldots$, correlated $\gamma_{2}-x_{1}$ using the NRTL equation 


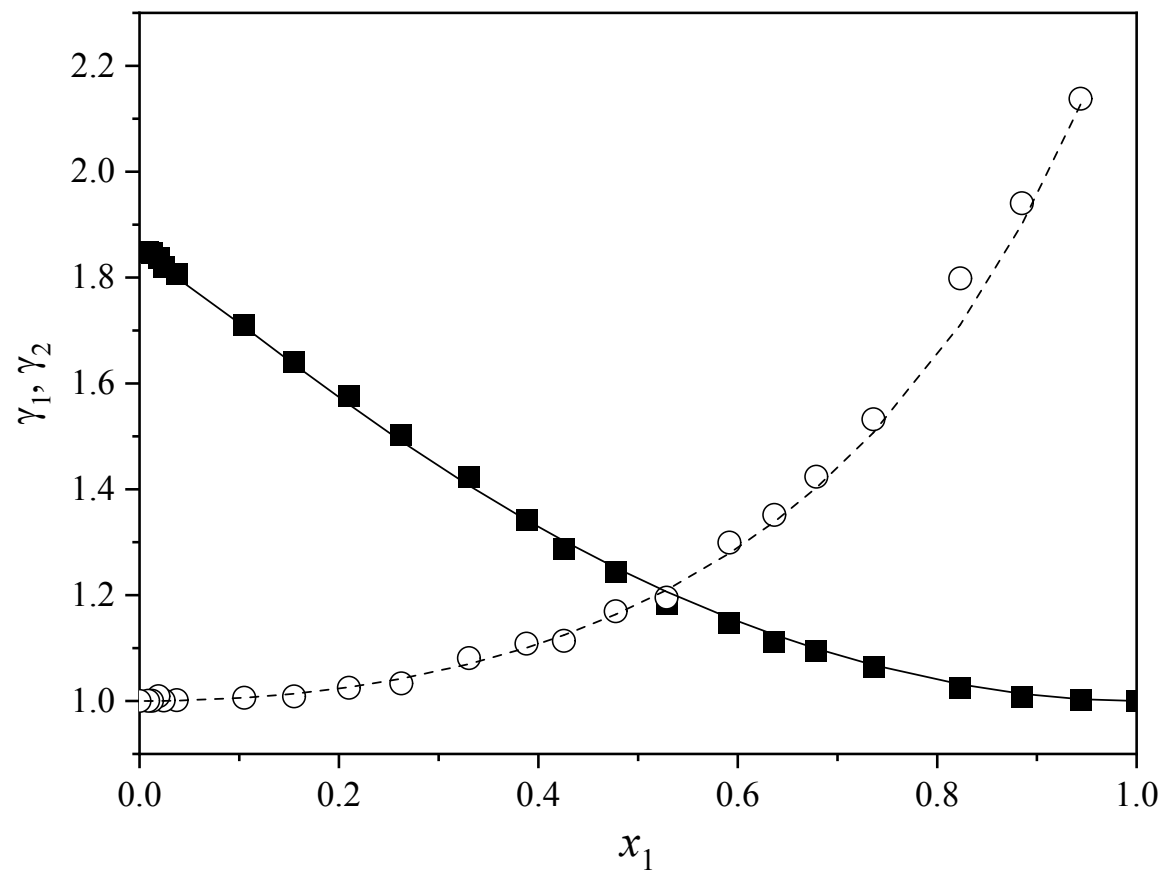

Figure S5. Plot of activity coefficients $\left(\gamma_{1}, \gamma_{2}\right)$ and molar fraction in liquid phase $\left(x_{1}\right)$ for the system $p$-xylene $(1)+$ ethyl benzoate (2) at $101.33 \mathrm{kPa}$ : $\mathbf{m}$, Experimental $\gamma_{1}-x_{1}$; - correlated $\gamma_{1}-x_{1}$ using the NRTL equation; $\circ$, Experimental $\gamma_{2}-x_{1} ; \ldots$, correlated $\gamma_{2}-x_{1}$ using the NRTL equation 